\title{
Are existing standard methods suitable for the evaluation of nanomedicines: some case studies
}

\author{
Sabrina Gioria*,1, Fanny Caputo ${ }^{2,3}$, Patricia Urbán ${ }^{1}$, Ciarán Manus Maguire ${ }^{4,5}$, Susanne \\ Bremer-Hoffmann ${ }^{1}$, Adriele Prina-Mello ${ }^{4,5}$, Luigi Calzolai ${ }^{1}$ \& Dora Mehn ${ }^{1}$ \\ ${ }^{1}$ European Commission, Joint Research Center (JRC), Directorate for Health, Consumers \& Reference Materials, Via Enrico Fermi \\ 2749, I-21027 Ispra, VA, Italy \\ ${ }^{2}$ Univ. Grenoble Alpes, F38000 Grenoble, France \\ ${ }^{3}$ CEA, LETI, Minatec Campus, F-38054 Grenoble, France \\ ${ }^{4}$ Laboratory for Biological Characterization of Advanced Materials (LBCAM), Department of Clinical Medicine, Trinity Translational \\ Medicine Institute (TTMI), School of Medicine, Trinity College Dublin, Dublin 8, Ireland \\ ${ }^{5}$ AMBER Center \& CRANN Institute, Trinity College Dublin, Dublin 2, Ireland \\ * Author for correspondence: Tel.: +39 033278 3584; Fax: +39 033278 5787; sabrina.gioria@ec.europa.eu
}

The use of nanotechnology in medical products has been demonstrated at laboratory scale, and many resulting nanomedicines are in the translational phase toward clinical applications, with global market trends indicating strong growth of the sector in the coming years. The translation of nanomedicines toward the clinic and subsequent commercialization may require the development of new or adaptation of existing standards to ensure the quality, safety and efficacy of such products. This work addresses some identified needs, and illustrates the shortcomings of currently used standardized methods when applied to medical-nanoparticles to assess particle size, drug loading, drug release and in vitro safety. Alternative physicochemical, and in vitro toxicology methods, with the potential to qualify as future standards supporting the evaluation of nanomedicine are provided.

First draft submitted: 2 November 2017; Accepted for publication: 13 December 2017; Published online: 30 January 2018

Keywords: analytical ultracentrifugation $\bullet$ cytotoxicity $\bullet$ drug release $\bullet$ field flow fractionation $\bullet$ high content screening $\bullet$ nanomedicine $\bullet$ nanoparticle tracking analysis $\bullet$ particle size distribution $\bullet$ regulatory $\bullet$ safety assessment

Despite a strong demand for fostering nanomedicine applications, as indicated by several analyses of global market trends, there is a general lack of specific protocols for their characterization at physicochemical and biological levels, which in a number of cases might become responsible for the failure in late clinical stage [1]. Hence, the rapid progress in the field of nanomedicines urgently requires the development of new standards or the adaptation of existing ones in order to assess the quality and safety of this emerging product class $[2,3]$. This is a prerequisite for the solid and safe industrial development of nanomedicines, a requirement of the public authorities or agencies funding R\&D in Nanomedicine, in both the USA and Europe.

A detailed understanding of the impact of the physical and/or chemical properties, and their association with clinical manifestations, is the focus of the biomedical research and pharmaceutical development. However, the challenge to identify critical physicochemical parameters of nanomedicines is increasing with the design of highly complex medical nanoparticles (Med-NPs) based on a wide variety of materials and chemicals. Nevertheless, there is a common consensus by regulators that some physicochemical parameters, including particle size distribution (PSD), chemical composition, drug loading and drug release kinetics are critical factors for the evaluation of the quality as well as for in vivo efficacy and safety of the nanomedicine formulations [4]. Currently, only a few standardized methods exist to characterize some of the crucial physicochemical properties and their impact on biological systems of Med-NPs. Unfortunately, they are often not applicable to the more complex and innovative nanoformulations. In addition, each product might require new or modified methods for its assessment. Since robust and validated measurement methods are essential for informed regulatory decisions on the quality and safety 


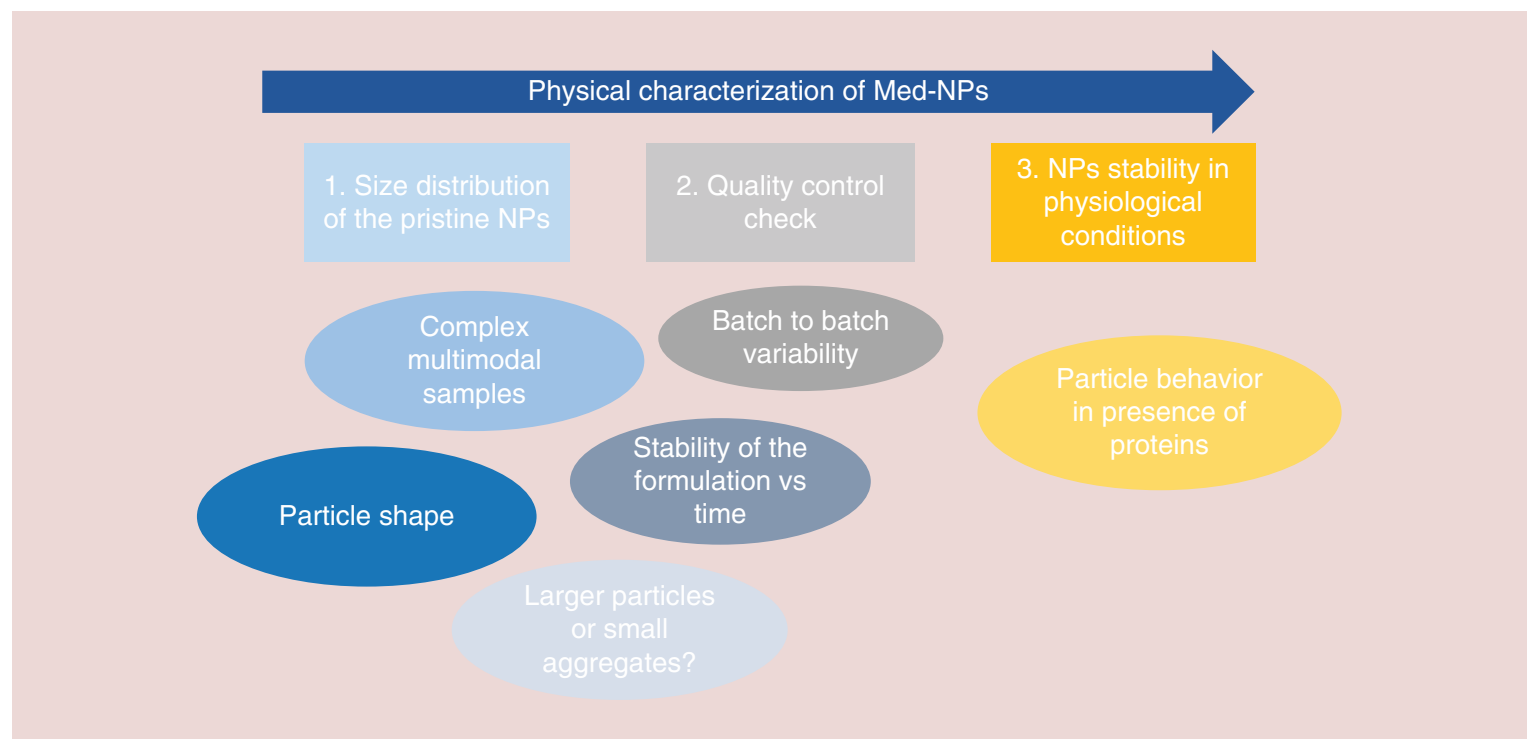

Figure 1. Importance of particle size and particle size distribution for the quality and safety of medical nanoparticles. The figure shows the importance of physical characterization of Med-NPs, to assess: (1) the particle size and shape of the pristine formulation and its stability, (2) the reproducibility of the manufacturing procedure and (3) the changes of size and shape of Med-NPs in physiological conditions.

Med-NP: Medical nanoparticle.

of Med-NP products, we have investigated the suitability of existing standards in selected case studies. Furthermore, we will provide some initial insight on robust physicochemical and in vitro toxicology alternative tests developed in the frame of the European Nanomedicine Characterization Laboratory (EU-NCL) H2020 project [5] that might qualify as future standards supporting the European regulators in the evaluation of nanomedicine candidates. The harmonization of standards, protocols and assays in the approval of new nanomedicine product is clearly a key priority as concluded at GSRS'16 [6].

The USA Nanotechnology Characterization Laboratory (NCI-NCL) and more recently the EU-NCL are already contributing to foster the use and deployment of standard operating procedures (SOPs) for Med-NP assessment. Further support is expected from the new H2020 project 'Regulatory Science Framework for Nano(bio)material-based Medical Products and Devices' (REFINE) launched in December 2017. In addition, a Community of Research in Nanomedicine between NNI in the USA (www.nano.gov/) and the European Commission in Europe has been proposed in September 2017 at the annual meeting of EU-USA Community of Researchers (https://us-eu.org/). The Community of Research is a flexible framework for EU-USA cooperation which includes regulatory agencies, scientific bodies and metrology institutes with the aim to work together for anticipating information needs and monitoring the progress in technological development to guide the harmonization of standards, protocols and assays for the approval of new nanomedicine products. This Community of Researchers in nanomedicine has highlighted the harmonization of protocols between scientists, regulators and standardization agencies from both USA and Europe, as its key priority.

\section{Physicochemical characterization: particle size distribution \& stability in biological media}

Particle size and size distribution are key factors for the manufacturing quality, as well as the efficacy and safety of Med-NPs, as shown in Figure $1[7,8]$. Average particle size and PSD are two of the most monitored parameters during the preclinical characterization of the Med-NPs, since they are known to impact the body absorption, biodistribution and excretion of the nanomaterials [9-11].

Dynamic light scattering (DLS) is the most widely used sizing technique [8] and one of the few methods applicable to the nanoscale range for which International Standards guidelines exist [12-14]. Despite the very common usage in the nanomedicine field, batch-mode DLS is generally considered a 'low-resolution' method [15-17] as it is subject to a strong dependence of scattering intensity on the particle size. It can be applied as a fast and simple method to check the integrity of monodispersed samples, determining the tendency of a nanoformulation to undergo significant 


\begin{tabular}{|c|c|c|c|}
\hline PSD of the pristine sample & $\begin{array}{l}\text { Only for monodispersed } \\
\text { samples [15-17] }\end{array}$ & Able to resolve complex PSD $[21,22]$ & Able to resolve complex PSD $[15,16,23,24]$ \\
\hline Particle shape & $\begin{array}{l}\text { No, only the hydrodynamic diameter of } \\
\text { an equivalent sphere is } \\
\text { calculated }[15,16]\end{array}$ & $\begin{array}{l}\text { No, only the hydrodynamic diameter of } \\
\text { an equivalent sphere is } \\
\text { calculated }[21,22,25]\end{array}$ & $\begin{array}{l}\text { Indirect information derived by the shape } \\
\qquad \rho=\frac{R_{g}}{R_{\mathrm{h}}[26,27]}\end{array}$ \\
\hline Batch to batch variability & $\begin{array}{l}\text { Not for heterogeneous samples or for } \\
\text { small changes }\end{array}$ & $\begin{array}{l}\text { Yes (internal quality control, data not } \\
\text { show) }\end{array}$ & Yes [28] (internal data not shown) \\
\hline Agglomeration/aggregation & $\begin{array}{l}\text { Not able to resolve dimers/multimers vs } \\
\text { pristine particles [16]. Useful as a quick } \\
\text { check for major agglomeration } \\
\text { phenomena }\end{array}$ & $\begin{array}{l}\text { Able to resolve the formation of } \\
\text { dimers/multimers [16] }\end{array}$ & $\begin{array}{l}\text { Able to resolve dimers/multimers. Able to } \\
\text { discriminate between dimers and larger } \\
\text { particles by their shape [16] }\end{array}$ \\
\hline
\end{tabular}

DLS: Dynamic light scattering; FFF: Field flow fractionation; MALS: Multi angle light scattering; Med-NP: Medical nanoparticle; NTA: Nanoparticle tracking analysis; PSD: Particle size distribution.

aggregation or degradation when exposed to conditions of high ionic strength, unsuitable $\mathrm{pH}$ or presence of proteins that may happen in physiological conditions [18]. However, it is not well suited for measuring the accurate PSD in case of polydispersed samples. DLS is unable to resolve the PSD of multimodal samples in similar size range $[15,17,19]$, it cannot detect the presence of small aggregates [16] and it is not able to resolve small modifications of the PSD happening under physiological conditions [20]. A schematic summary of the limitations of batch mode DLS in the physical characterization of Med-NPs, and the potential of two alternative techniques, nanoparticle tracking analysis (NTA) and field flow fractionation (FFF) coupled with online sizing detectors (DLS-multi angle light scattering [MALS]) is described in Table 1.

DLS's companion technique, NTA, is also becoming one of the standard approaches for the characterization of particles in suspension. Similar to DLS, it has also been developed into American Society for Testing and Materials (ASTM) [32] and International Organization for Standardization (ISO) [33] standards, and operates under the basis of light scattering and Brownian motion like DLS. Unlike DLS, however, the NTA system and its proprietary software tracks individual particle movements to calculate the diffusion coefficient for each individual particle. As such it is a high-resolution analysis technique that is able to distinguish small differences between two particles or populations, either based on diffusion and Brownian motion, or light scattering intensity.

One crucial advantage that NTA has over DLS is that it is not biased toward larger particles or aggregates. The NTA software is based on the tracking of single particles, whereas typical DLS techniques place a strong bias on the largest particles present in the sample [34]. This allows for the detection of secondary peaks, which may not be detectable using other traditional measurements. The counting of individual particles also enables the concentration of the sample in nanoparticles per milliliter $(\mathrm{NPs} / \mathrm{ml})$, to be simultaneously determined as the volume of the field of view is known.

The recent incorporation of the finite track length adjustment algorithm has advanced the analysis of polydisperse and multimodal samples by improving size distribution peak isolation and resolution [21,22]. Finite track length adjustment algorithm accounts for the tracking of a particle over a finite number of frames leading to a statistical error in the average particle diameter [22]. Where a polydisperse sample is analyzed using DLS, the Z-average or cumulants mean will consist of one value only, weighted toward the largest component. In the case of NTA, the software will be able to resolve the multiple components of the sample as previously shown [21].

However, there are limitations of the technique. While the visualization of particle light scattering is a distinct advantage for NTA over other techniques, the dynamic range of the camera and the laser setup can introduce the opportunity for measurement errors and inaccuracies. Under Rayleigh and Mei scattering, shorter wavelengths of light scatter more strongly than longer wavelength, with a shorter wavelength being required to visualize smaller NPs. The use of a short wavelength laser in NTA setups, such as the $405 \mathrm{~nm}$ laser, can allow for the detection of the smaller components of a polydisperse sample. However, this can cause the larger particles to become overexposed 
leading to the small particles becoming obscured. The light scattering potential of the particles also plays a role in accurate concentration measurements, as it does with size determination. Particles with low refractive indices will inherently result in more uncertain results due to the system's operation limits.

To overcome the inherent difficulties that are imposed when analyzing a polydisperse sample by DLS or NTA, a fractionation step can be incorporated prior to measurement. This can be done by using DLS and MALS detectors coupled online to systems such as the asymmetric flow FFF (AF4) or size exclusion chromatography.

AF4 is a robust method based on the generation of a parabolic flow profile in a narrow ribbon-like channel, via the application of a liquid cross-flow, without the need of a stationary phase [26,27,35]. This versatile and gentle fractionation technique was successfully used to sort different types of Med-NPs, including liposomes, lipid NPs, polymeric NPs, virus like particles, metallic NPs and metal oxides, allowing for the characterization of their pristine properties, stability and behavior in a biologically relevant environment as recently reviewed by many authors $[27,36,37]$. AF4 NP-sorting coupled to online DLS and/or MALS allows to resolve the PSD of very complex samples and to analyze small changes in particle size, which are both very important aspects for quality control and regulatory aspects of the approval of the nanomedical products. Interestingly, by AF4-MALS-DLS, it is possible to directly obtain information about the particle shape (e.g., spherical vs elongated particles) by calculating the shape ratio $\rho=\frac{R_{g}}{R_{h}}$, which is the ratio of the radius of gyration ( $\left.\mathrm{Rg}\right)$ determined by MALS and the hydrodynamic radius measured (Rh) by DLS [27,36].

The NCI-NCL and EU-NCL have developed an SOP to determine NP size distribution by coupling AF4 with on-line size measurement (DLS and/or MALS), obtaining the very powerful combination of AF4-MALSDLS. This technique applicable to Med-NPs might be considered as alternative to the DLS alone for regulatory purposes [30]. This SOP was successfully applied in the EU-NCL consortium to characterize pristine Med-NPs with the aim to (i) resolve the PSD of complex mixtures, (ii) discriminate between population of particles with different shapes, (iii) discriminate between larger particles and small aggregates, and (iv) to monitor small changes of PSD due to instances like batch to batch variability or instability during long-term storage, all cases where batch-mode DLS fails [15-17]. In addition, EU-NCL has also developed an SOP to study the NP behavior in presence of serum proteins, which is crucial to understand the biological effects of Med-NPs in vitro and in vivo [38].

Doxil $^{\circledR}$ (the first nanomedicine to be approved by US FDA in 1995) is a PEGylated nanoliposome, currently used to treat ovarian cancer [39]. In terms of PSD, Doxil is a perfect example of a good monodispersed sample: batch mode DLS, NTA, FFF-MALS-DLS and cryo-transmission electron microscopy (TEM) measurements (Figure 2), all detect the presence of a single population of spherical particles in the range of 40-90 nm. In such an ideal case, a batch mode DLS analysis will be sufficient to measure the real PSD of the sample. However, as already shown by many studies $[15-17,20]$, this is not always true for more heterogeneous samples, which are characterized by multiple populations with different sizes and/or shapes.

Typical examples of more heterogeneous niosome (nonionic surfactant vesicle) and polymeric NPs were presented by Ingallina et al. [24] and Varenne et al. [15]. The analysis of the samples with AF4-MALS-DLS and NTA indicates the presence of a large heterogeneous population of particles of different sizes. In the polymeric formulation, the FFF-DLS and NTA can resolve the presence of four distinct populations of particles which differs in size, while according to batch mode DLS, the sample was nicely monodispersed.

Iavicoli et al. demonstrated the potential of AF4-DLS-MALS to study subtle changes in PSD and morphology of anionic liposomal formulations interacting with positively charged antimicrobial peptides [23]. Using online sizing detectors, it was possible to detect the formation of an NP-peptide complex with a larger size and a perturbed morphology in addition to the pristine population of unilamellar vesicles. Thanks to the calculation of the shape ratio, it was also possible to determine the multilamellar phase of the larger population, induced by the bridging effect of the positively charged peptide on the negatively charged membrane of the liposomes. Not surprisingly, batch mode DLS was not able to resolve the presence of two populations in the peptide-NP mixture, and only detected one larger population shifted to larger sizes.

The examples presented, among many others [17,19], demonstrated that when PSD and morphology of heterogeneous samples are studied, batch mode DLS analysis provides somewhat misleading results, while the FFF-MALSDLS and the NTA are able to resolve the real PSD of the sample in very complex samples. Thanks to the coupling of online DLS and MALS detectors, FFF-MALS-DLS can also give information about particle shape (see Table 1). 


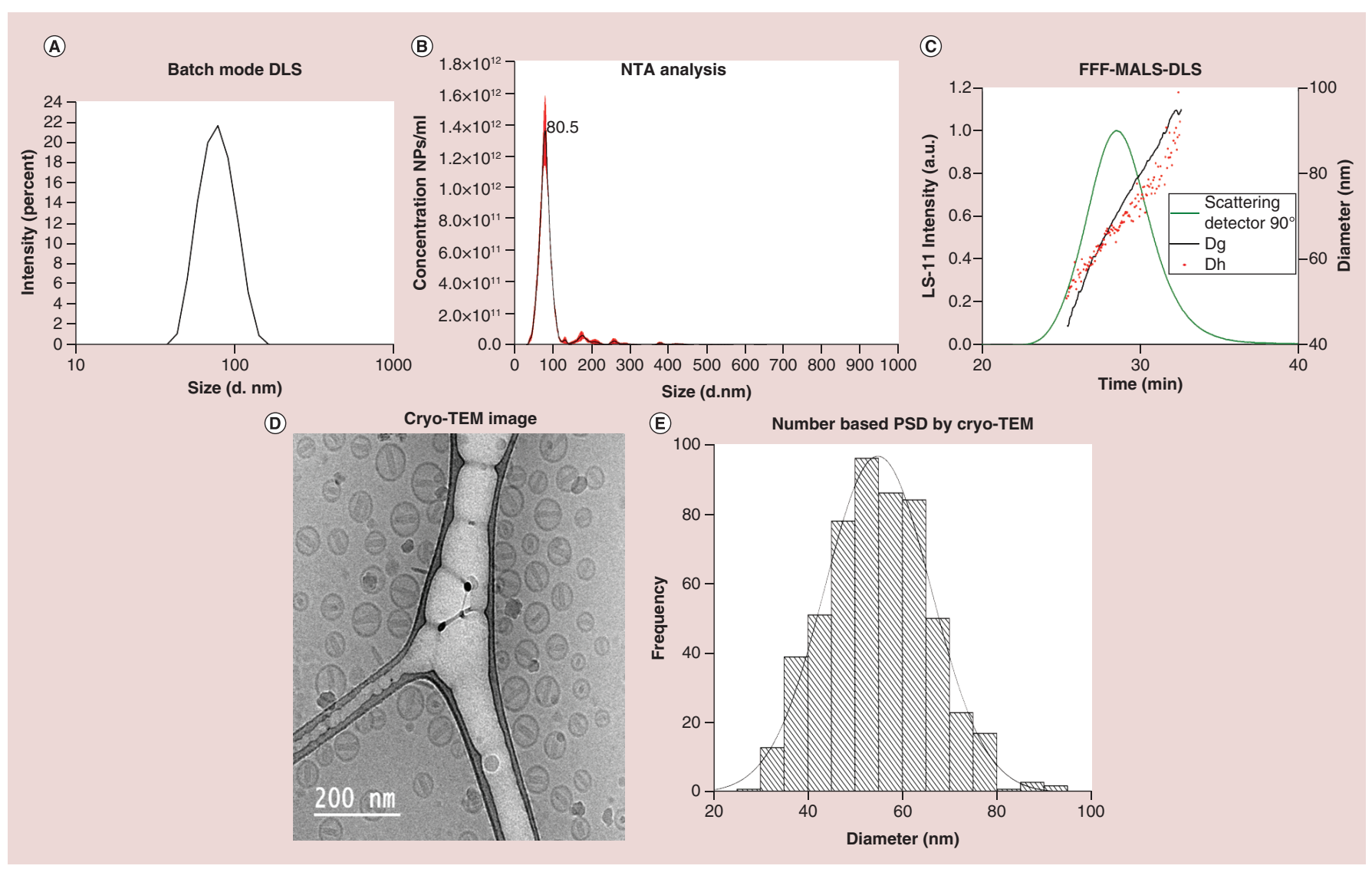

Figure 2. Characterization of Dox-NPTM liposomes. (A) Batch DLS, intensity-based size distribution liposomes at $100 \times$ dilution in PBS. Average of ten measurements. (B) NTA analysis at 10,000 $\times$ dilution in PBS. Average \pm SEM of $6 \times 60 \mathrm{~s}$ videos. (C) FFF elugram (absorbance) peak (green), hydrodynamic diameter by DLS (red) and geometrical diameter by MALS (black). (D) Cryo-TEM images and (E) corresponding PSD (diameter of an equivalent sphere). Solid line: Fit of the PSD by a Gaussian distribution. All the data clearly indicate a well-monodispersed sample characterized by one population.

DLS: Dynamic light scattering; FFF: Field flow fractionation; MALS: Multi angle light scattering; NP: Nanoparticle; NTA: Nanoparticle tracking analysis; PBS: Phosphate-buffered saline; PSD: Particle size distribution; SEM: Standard error of mean; TEM: Transmission electron microscopy.

When looking at multimodal particle distributions, it is important to understand if the bigger sizes are associated with larger single particles or with small aggregates. A recent publication by Mehn et al. [16] showed that the combination of AF4 and TEM is a useful strategy to uncover dimers and multimers of NPs, where batch mode DLS completely fails. In this work, the single particles and the dimers were fractionated by AF4, PSD was measured by DLS and MALS, the different fractions were collected after the AF4 run and at last, visualized by TEM. The introduction of the online separation prior to the size measurement by DLS and MALS allowed more accurate size information and the calculation of the gyration/hydrodynamic radius ratio gave a good indication of the shape of the particles, suggesting the presence of a nonspherical population associated to dimers. The final confirmation of the presence of dimers came from direct visualization by TEM of the different population collected after the AF4 fractionation.

One of the crucial factors for the successful translation of Med-NPs is the ability to produce multiple reproducible batches, having the same properties, including the PSD. Batch to batch variability has to be controlled at the early stages of development of Med-NPs, since significant variation of the PSD between batches can result in different biological effects, as shown by Schädlich et al. [28]. Low resolution batch mode DLS is often not suitable for quality control purposes to detect small changes of size in different batches (unpublished data not shown). AF4-MALS-DLS allows resolving slight differences in PSD between batches, being a promising alternative technique to be adopted for quality control in routine [28]. The high-resolution nature of NTA also allows for the analysis of complex samples, as previously shown, as well as the detection of components that may be present in small concentrations [21,25]. 
The NTA resolved quality and its validated accuracy and reproducibility may be another suitable option for quality control purposes to detect small differences in batch sizes, as shown by Maguire et al. [21].

Intravenously administered Med-NPs are generally designed to avoid interaction with blood components, usually by PEGylation, since protein adsorption from blood induces aggregation or destabilization of the formulation, influences their fate and circulation time and thus, drastically modulates the in vitro and in vivo responses. For these reasons, investigating the interaction of Med-NPs incubated in cell culture media and/or with plasma proteins should be crucial for the regulatory approval. The protein adsorption on NPs can be studied by the change in NP size after incubation in the presence of serum proteins. Unfortunately, the protein corona is practically invisible for imaging methods like TEM, and it might become impossible to properly quantify with batch mode DLS in biologically relevant medium [20]. Similarly, the analysis of such samples by NTA would also yield questionable data as the refractive index (RI) of the particles so closely matches that of the solvent, impeding accurate characterization. In this context, FFF-MALS-DLS can provide accurate size information for polydispersed samples in physiological media and for protein binding studies by fractionating the free protein and the NPs in the media before sizing the Med-NPs.

The batch mode DLS analysis and the FFF-MALS-DLS of lipid NPs [40] and of Doxil NPs [39] before and after incubation with serum proteins performed according to the SOP developed by the EU-NCL are shown in Figure 3. While no significant differences are detected for the average particle size of lipid NPs when in contact with serum proteins (Figure 3A \& B), batch mode DLS analysis of Doxil NPs after incubation with serum is slightly shifted to smaller size (Figure 3C). In the latter case, based on a single DLS experiment, it is not possible to determine if the shift in PSD is caused by a small, real change of the PSD of the NPs, if the results are biased because of viscosity and multiple light scattering phenomena in the presence of ions and proteins or if the mathematical analysis provides a distorted solution without resolving the contribution of the signal by serum proteins (smaller size) with that by the liposome population (larger size).

The comparison of elution profiles registered using the light scattering detector online coupled to the AF 4 channel helps to find the answer. As shown in Figure 3D, in the presence (red) or absence (black) of serum proteins, the AF4-MALS-DLS elution profile and the PSD of both samples are overlapping, suggesting negligible NP-protein interactions and a misleading shift detected by batch mode DLS. The stability of the two Med-NPs analyzed is not surprising due to the PEGylation on their surface. However, not all the Med-NPs are stable in the presence of serum proteins: for example [31], showed by AF4-MALS, the partial or total disassembly of polymeric micelles after a few hours of incubation with serum proteins. Necessarily, in formulations like these, stability has a dominant effect on drug release kinetics, linking the modification of the particle size, for example, particle degradation, to the analysis of drug release.

AF4-MALS-DLS possess a great potential in the characterization of Med-NPs, being a powerful and robust analytical technique that allows overcoming the limitations of the batch mode light scattering sizing techniques. It allows to resolve the PSD of complex samples and to analyze slight changes in the PSD, often preventing the misinterpretation of the results resulting from batch mode DLS analysis.

Nevertheless, also AF4 has some disadvantages: the ideal elution conditions for each Med-NPs are different and method optimization can be very laborious. It is recommended to follow an SOP which includes some critical quality checks to be aware of the possible sample loss in the channel, and to ensure appropriate analysis of the data obtained by light scattering $[27,30,36,37]$ Moreover, it is often necessary to compare the results with orthogonal highresolution techniques, such as the direct visualization of the Med-NPs by electron microscopy. However, when the elution method for specific Med-NPs is successfully developed and if a robust SOP is followed, AF4-MALS-DLS is a promising tool for synthesis optimization, quality control and to monitor the stability of the Med-NPs.

\section{Drug loading/drug release kinetics}

Another major regulatory concern is the quantitative determination of free and bound/encapsulated drug, in addition to the total drug content $[41,42]$. In fact, it can be generally expected that the NP-bound drug fraction will show different pharmacokinetic behavior and biodistribution than the free drug fraction. Accordingly, the fraction of encapsulated active substance fraction is listed among the parameters to be addressed in the submission of liposomal products in the reflection paper released by the EMA [42]. Regarding the applicable analytical methods, this European directive refers to the European pharmacopeia monographs (and in case of nonavailability, member state monographs) specific for the individual active substances [43]. In general, both the European regulators and 
(A) Lipid nanoparticles: Batch mode DLS

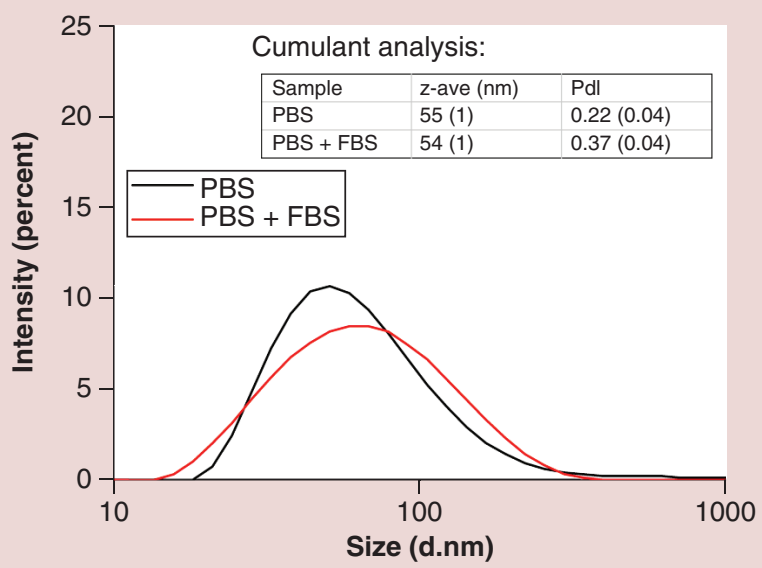

(C)

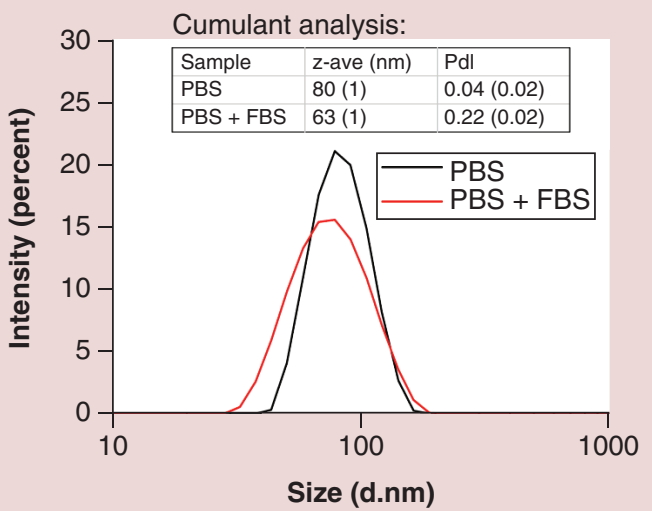

(B)

Lipid nanoparticles: FFF-DLS MALS

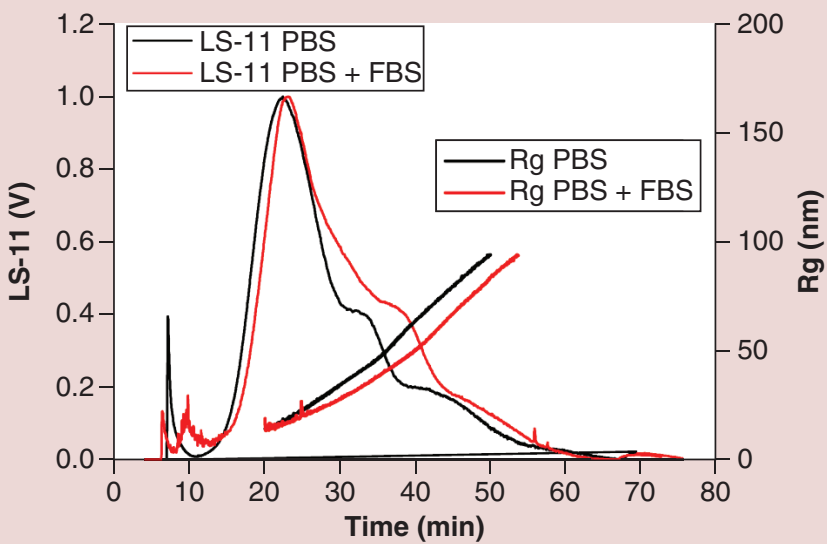

(D)

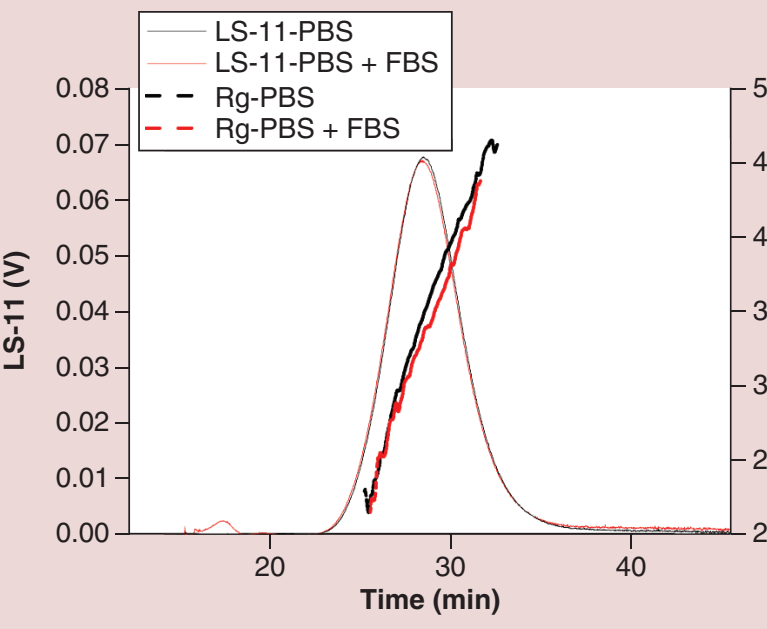

Figure 3. Stability of lipid nanoparticles and Dox-NP ${ }^{T M}$ liposomes and lipid nanoparticles in serum. Pristine particles are represented in black and particles incubated with serum in red. (A) PSD and cumulant analysis by batch mode DLS of Lipidots diluted 10x in PBS and in the presence of serum proteins. (B) FFF-MALS analysis of the Lipidots diluted $10 \times$ in PBS with and without serum proteins. No major differences are detected in the analysis in the absence or presence of serum proteins. (C) PSD and cumulant analysis by batch DLS, intensity-based size distribution liposomes. (D) FFF-MALS elugram and geometric radius (Rg) by MALS. Batch mode DLS of Doxil ${ }^{\circledR}$ NPs indicates a small shift of the PSD to smaller sizes while according to FFF online DLS, the PSD is not influenced by the presence of serum proteins.

DLS: Dynamic light scattering; FFF: Field flow fractionation; MALS: Multi angle light scattering; NP: Nanoparticle; PBS: Phosphate-buffered saline; PSD: Particle size distribution.

FDA request the documentation of rigorous method validation for analytical procedures used in the characterization of the medicinal products [44,45].

The measurement of the free and bound drug fractions has been addressed by using drug-specific and nonspecific analytical methods. Determination of the total drug content is usually based on the disruption (by freeze-drying, surfactant, organic solvent) of the NPs and subsequent quantification of the drug (or active ingredient) by using HPLC coupled with appropriate detectors (UV-Vis or fluorescence) [46,47]. The HPLC method is substance specific: it is able not only to quantify the active substance, but also to identify it (based on its retention time) and distinguish from possible impurities. Typically, methods to measure free versus encapsulated drug ratio include a separation step, ultrafiltration, ultracentrifugation, solid-phase extraction, size exclusion, AF4 and liquid chromatography and a separate analysis step using substance-specific liquid chromatography methods combined with various detection techniques (UV-Vis, fluorescence, charge aereosol detector, mass spectroscopy) [46-51]. The limitation of this methodology is that filtration devices and centrifuge tubes might adsorb the analyte or even the nanoformulated 


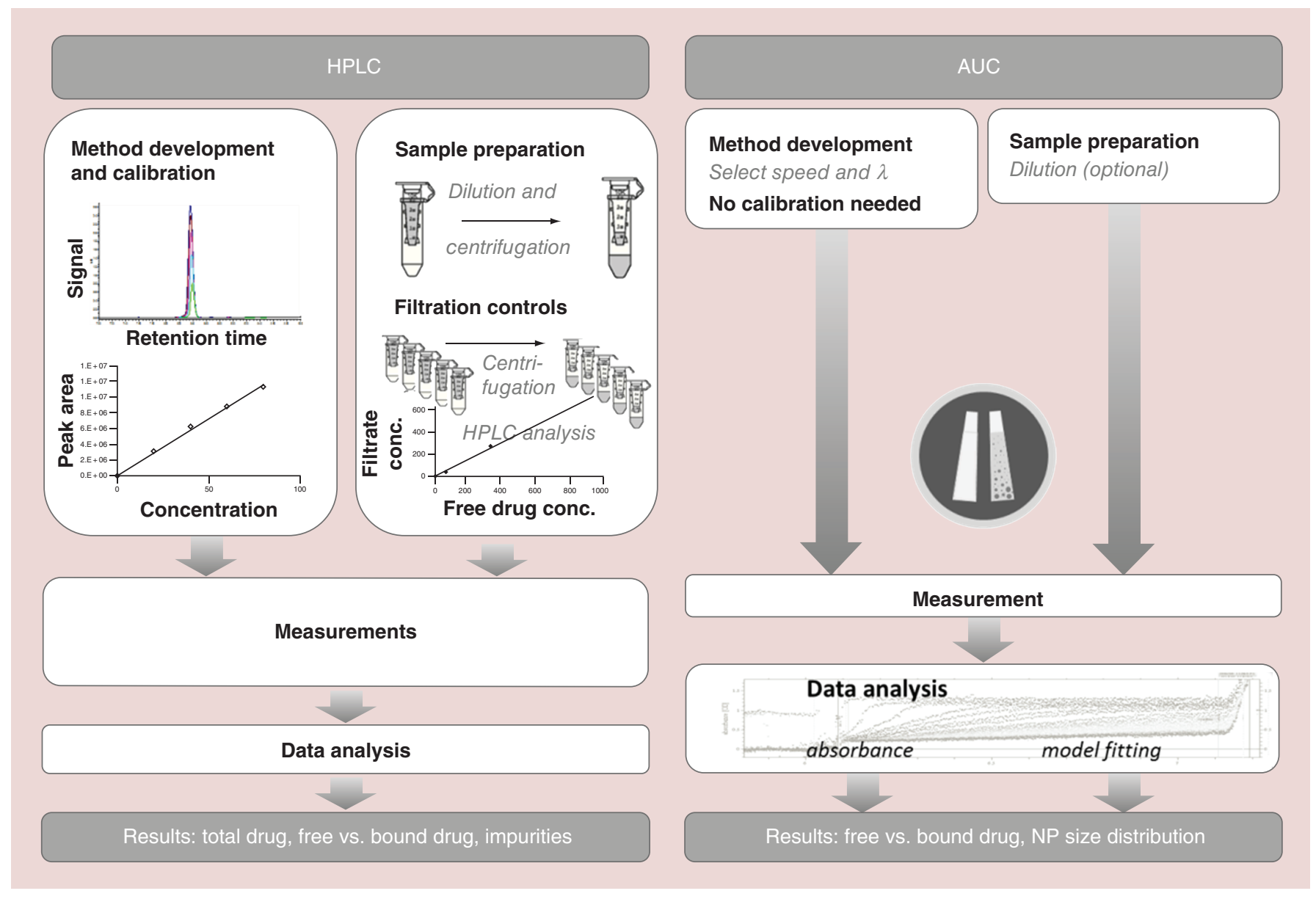

Figure 4. Illustration of measurement complexity, invested experimental times and resulting information in case of HPLC and analytical ultracentrifugation (AUC)-based free versus bound drug analysis.

AUC: Analytical ultracentrifugation.

product depending on size, surface charge and the buffer applied. In addition, dilution before separation might provoke drug release. Because of these reasons, careful method development and a series of well-designed control measurements are necessary, resulting in increase of analysis time and complexity (Figure 4).

As an alternative, analytical ultracentrifugation (AUC) has been shown to be a fast and simple method for determining both the PSD and the free/encapsulated drug ratio [52]. In fact, AUC combines separation, concentration and detection steps into one single measurement improving total analysis times and reducing experiment complexity (Figure 4).

The detection modes of AUC are not substance specific, though the method has other advantages. It is a technique originally developed for biomolecule (protein) mass measurements and kinetic studies [53-55] but it is also capable to analyze size distributions of NPs of diverse nature - depending on their density - up to the micrometer range [56,57]. AUC measurements do not need calibration using size standards, as it is a first-principles-based technique that calculates particle diameter from the sedimentation coefficient (i.e., speed) of the components in suspension $[58,59]$. Nowadays, state-of-the-art AUC instruments include both absorbance and/or RI detectors that allow monitoring the sedimentation of polymer-based NPs or liposomes in aqueous suspensions simultaneously using both optics. The measured signal change in time is used to calculate sedimentation coefficient (s) distributions by applying various models, and then $s$ distributions can be (in case of RI optics directly) converted to mass metrics size distributions. Without known particle densities (which are often the case for complex structure Med-NPs), direct contrasting of size distributions can still carry information on interbatch variability or drug loading. In typical Med-NPs, the molecular weight of the active ingredient is about five orders of magnitude lower than the weight of the complete Med-NP, therefore the sedimentation of the particles is much faster than the sedimentation of the unbound active 


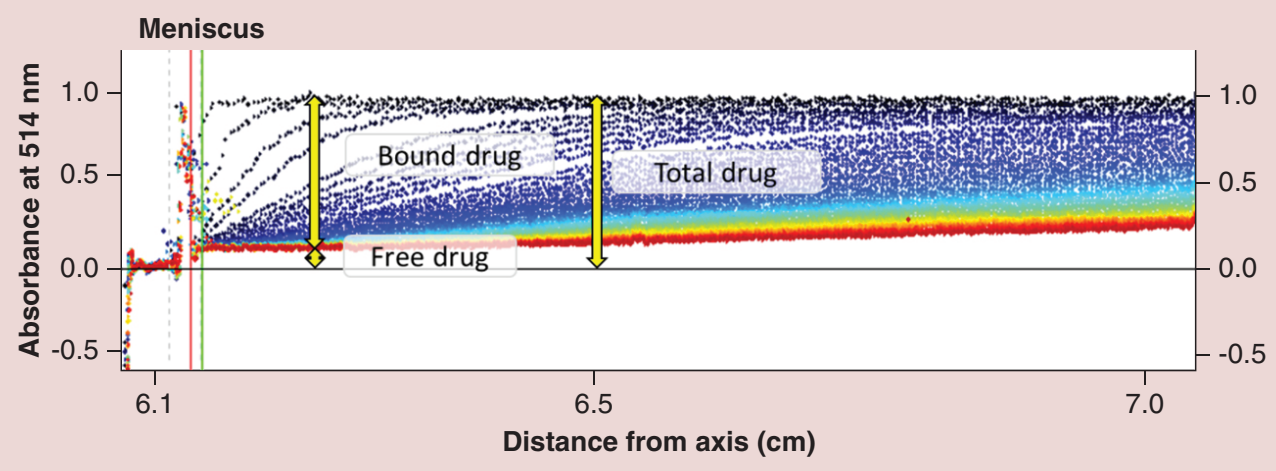

Figure 5. Analytical ultracentrifugation absorbance data of daunorubicin-loaded liposomes registered at $514 \mathrm{~nm}$ showing sedimentation of nanoparticle-bound drug and the presence of about $12.3 \%$ nonsedimenting free drug. Colors (in electronic version) represent various time points from the beginning (blue) to the end (red) of the measurement.

compound. For drugs with a well detectable and specific UV-Vis absorption profile, at well-selected centrifugal force, this difference results in a practically stable time-independent and radius-independent background. A typical example of AUC absorption-based sedimentation detection is shown in Figure 5 for daunorubicin-loaded liposomes. The ratio of the residual signal of the unbound drug registered after the sedimentation of larger particles can be compared with the absorption corresponding to the fast sedimenting component. This allows a fast concentration ratio estimation for the unbound and Med-NP-bound active ingredient. Additionally, AUC also needs negligible method development procedure and no calibration compared with HPLC (Figure 4).

Despite the obvious advantages described here, characterization techniques like AUC might still require some time before becoming widely used, validated and accepted by regulatory bodies. Among the assays recommended for the analysis of bulk drug materials, substance-specific methods such as HPLC and gas chromatography (GC) have dominated in the US pharmacopeia for the past 10 years, and the ratio of chromatographic procedures has also systematically increased in the European pharmacopeia [60]. Still, nonspecific analytical techniques like AUC might become powerful tools in tackling new characterization challenges linked to the introduction of complex Med-NPs.

\section{In vitro toxicity}

An important aspect when assessing the safety of a nanoformulation is the evaluation of its toxicity. In vitro methods are often based on human cells $[61,62]$ and can support the elucidation of the mode of action of adverse effect in humans, in particular, when combined with advanced methodologies such as omics or new imaging technologies [6367]. In addition, in vitro cytotoxicity testing is an integral part for the identification of the hazardous potential of drug candidates in the early phase of drug development providing initial insights in the toxic potential of a formulation at low costs and in a short timeframe. For nanomedicine development, in vitro tests are playing a multiple role providing indication on the toxic potential of the formulation, and in revealing toxic residuals resulting from the manufacturing process and can give an indication of the stability of the drug delivery system [68]. As such, in vitro methods are very attractive tools for product developers especially, as said, in the early phase of the development.

Similarly, predictive in vitro methods are an essential part of the 3Rs (Replacement, Reduction and Refinement) principle [69]. Thus, the availability of in vitro methods that are fit for their application in biomedical research and in the preclinical assessments of Med-NPs contributes to the 3Rs-EU policy objectives [69-71].

Safety data for medical devices or nanoparticulate materials can be obtained by testing in accordance to guidelines published by international standards development organizations such as the ISO [72] or the ASTM [73]. A limitation to the applicability of the standardized in vitro test methods for Med-NP assessment is the possible interference of the nanomaterial with the test reagents or at the assay readout that can lead to false predictions as reported in several publications [68,74-80]. Similarly, the recent released ISO/TR10993-22:2017 (E), which provides guidelines specific for nanomaterials, states that the various assays described in the ISO 10993 series are not always appropriate in the testing of nanomaterials [81]. The need to overcome current pitfalls in toxicological assessments has also been 


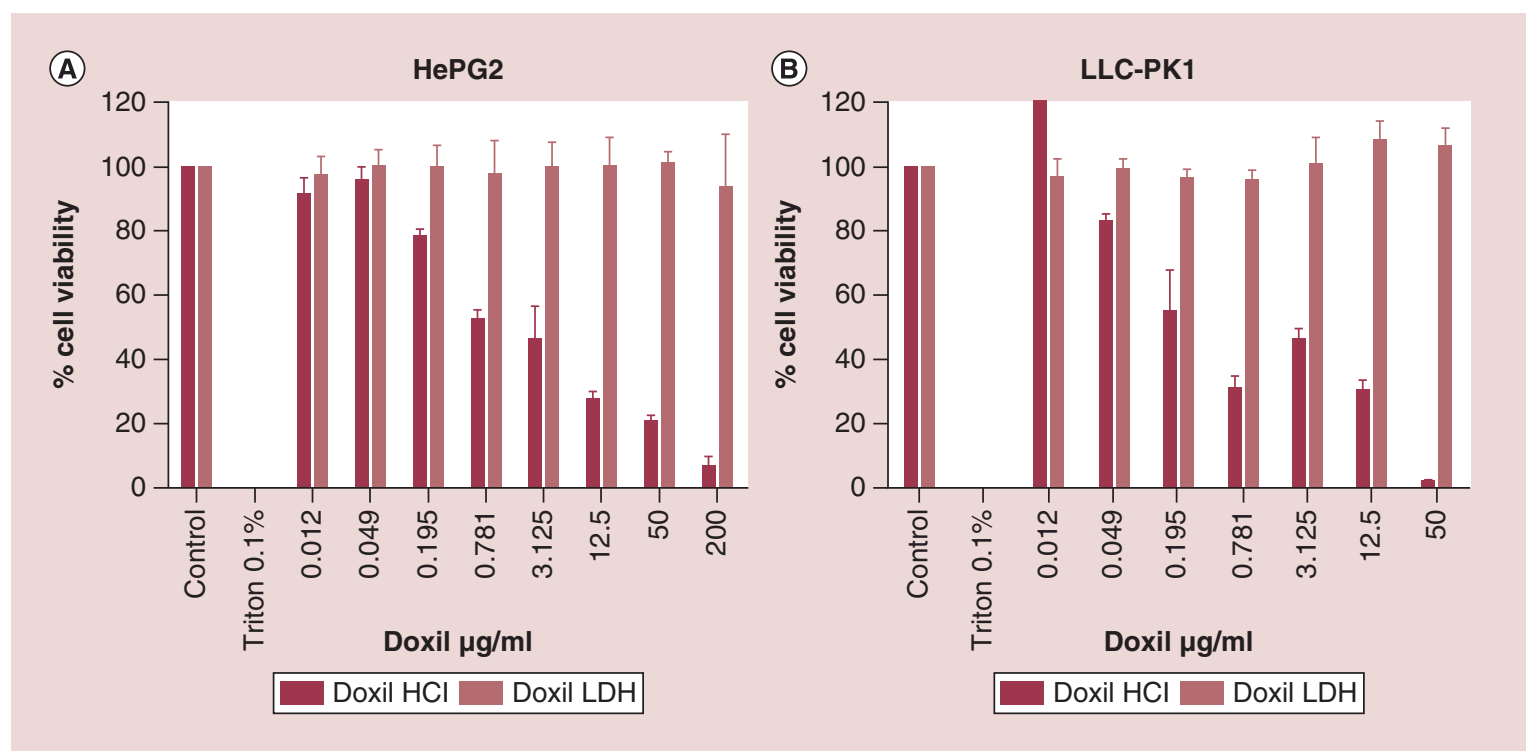

Figure 6. Comparison of cell viability data obtained by lactate dehydrogenase and high content screening. LLC-PK1 and HePG2 have been exposed to Doxil ${ }^{\circledR}$ for $48 \mathrm{~h}$. Results obtained by LDH assay are here compared with the results from HCS, the latter used to assess cell viability via Hoechst/PI staining. Data clearly indicate that LDH is not able to assess Doxil toxicity and to provide a dose-response curve in both the cell lines used. In contrast, results obtained by HCS indicate that HCS allows for the assessment of toxicity, and the provision of dose-response data. Data are reported as percentage of cell viability with respect to the untreated cells. Triton $0.1 \%$ has been included as positive control. Three biological replicates have been performed.

HCS: High content screening; LDH: Lactate dehydrogenase; PI: Propidium iodide.

highlighted in a recent survey organized by the Joint Research Center of the European Commission with the International Pharmaceutical Regulatory Forum in the frame of the H2020 EU-NCL project [82].

According to ISO 10993-5:2009 and ASTM E2526-08, lactate dehydrogenase (LDH) release and 3-(4,5Dimethylthiazolyl-2)-2,5-diphenyltetrazolium bromide (MTT) reduction are standard methods to address the biological evaluation of medical devices and nanoparticulate materials. Here, we present the limitations of the LDH assay when applied to assess Doxil in vitro cytotoxicity on the two cell lines LLCPK1 and HepG2, and also provide an alternative method.

In Figure 6, data obtained by LDH are confronted with results obtained by high content screening (HCS), which has been used to assess cell viability via Hoechst/propidium iodide (PI) staining, a noncolorimetric assay. The data obtained clearly indicated that $\mathrm{LDH}$ is not able to provide dose-response curve to assess the toxicity of Doxil in any of the two cell lines evaluated. In contrast, results obtained by HCS indicate that HCS allows for more correct toxicity assessment, as well as providing dose-response relations.

Another example of limitation of the current standardized methods for in vitro cytotoxicity assessment when applied to Med-NPs is reported in Figure 7. By using LDH release assay, the result obtained for LLCPK1 cells at the highest tested dose of $105 \mu \mathrm{g} / \mathrm{ml}$ is clearly misleading. Morphological characterization by phase contrast microscopy of LLCPK1 cells exposed to $105 \mu \mathrm{g} / \mathrm{ml}$ of polyvinylpyrrolidone (PVP)-AgNPs shows irregularity in cell shape, specifically cell shrinkage and extensive cellular detachment, confirming that the data obtained by LDH assay must be interpreted carefully. Incompatibility of AgNPs with LDH assay has already been reported in literature [83]. When assessing cell viability via Hoechst/PI staining and HCS, no limitations in the response were observed. Detection of Hoechst/PI staining can be accomplished not only by HCI, but also other methods, including FACS.

The MTT assay is another standardized colorimetric test used to address the biological evaluation of medical devices in terms of in vitro cytotoxicity according to ISO 10993-5:2009, or for the evaluation of nanoparticulate materials following the ASTM E2526 - 08 (2013). However, there are also constraints in its applicability when used to screen Med-NPs, due to the possible interferences of the NPs with the assay component, and in the reading of the absorbance of the colorimetric assay. Again this has been reported in several publications $[84,85]$ and in the most recent ISO/TR 10993-22 [81]. 
Figure 7. Comparison of cytotoxicity data obtained by lactate dehydrogenase and high content screening. LLC-PK1 cells have been exposed to PVP-coated AgNPs-PVP for $48 \mathrm{~h}$. Results obtained by LDH assay, an ISO and ASTM standardized method, are compared with results obtained by HCS, assessing cell viability via Hoechst/PI staining. The data obtained by the LDH release assay, for LLC-PK1 cells at the highest dose tested of $105 \mu \mathrm{g} / \mathrm{ml}$, are clearly misleading and not in agreement with morphological observation. When assessing cell viability via Hoechst/PI staining and HCS, a more accurate reflection of cellular toxicity is observed, particularly at higher concentrations. Data are reported as percentage of cell death with respect to the untreated cells $(n=3)$.

ASTM: American Society for Testing and Material; HCS: High content screening; ISO: International Organization for Standardization; LDH: Lactate dehydrogenase; NP: Nanoparticle; PI: Propidium iodide; PVP: Polyvinylpyrrolidone.

Figure 8. $\quad$ Doxil ${ }^{\circledR}$ cytotoxicity data by MTT assay. LLC-PK 1 cells exposed to Doxil for $48 \mathrm{~h}$. The graph shows that at concentrations greater than $12.5 \mu \mathrm{g} / \mathrm{ml}$, the dose-response curve shows an unusual trend. More specifically, as the dose increases, there is a decrease in metabolic activity, as typically expected, until this critical point is reached. However, at concentrations above $12.5 \mu \mathrm{g} / \mathrm{ml}$, data obtained show an increased metabolic activity. Data are expressed as percentage of metabolic activity with respect to the negative control. Triton $0.1 \%$ has been included as positive control $(n=3)$.

MTT: 3-(4,5-Dimethylthiazolyl-2)-2,5-diphenyltetrazolium bromide.
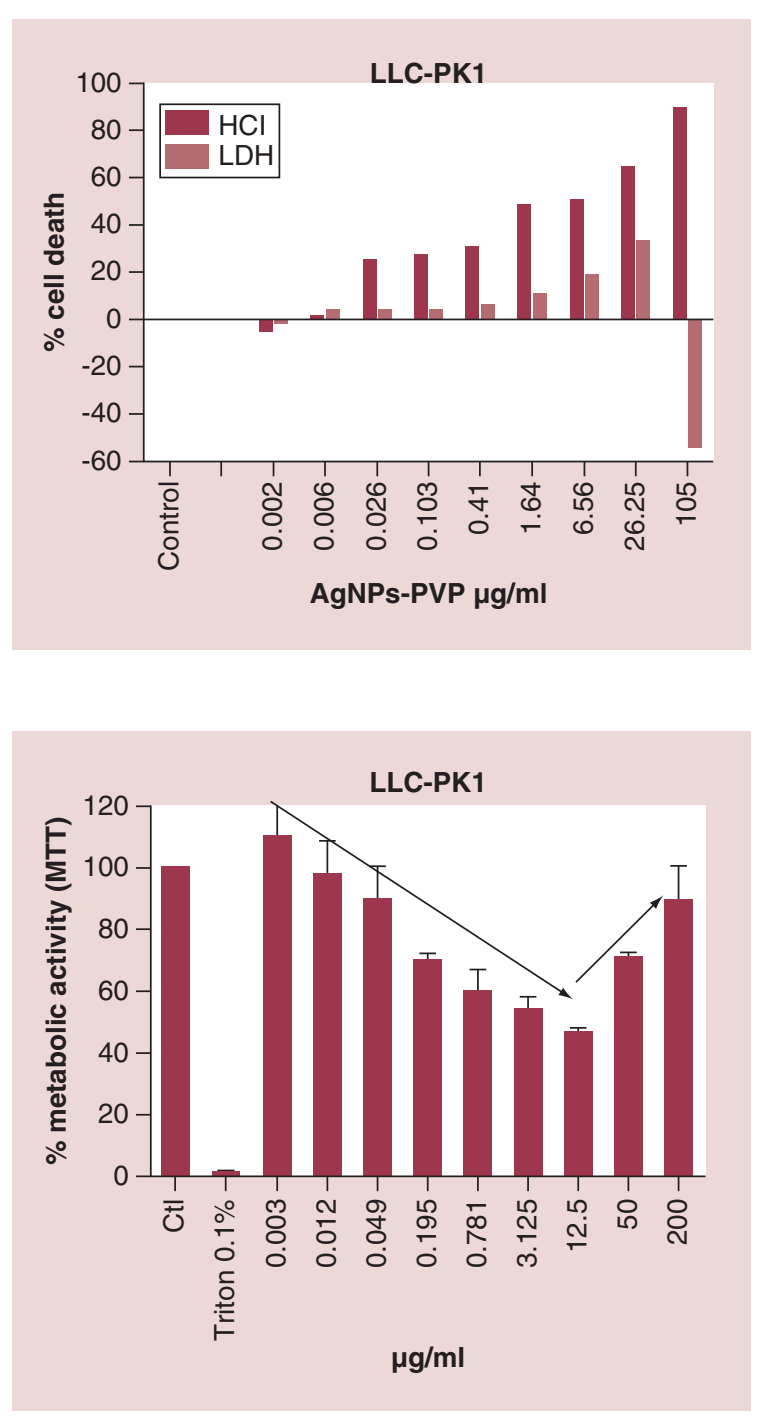

For instance, we have found that when assessing Doxil toxicity by MTT assay in LLCPK-1 cells for $48 \mathrm{~h}$, at concentrations above $12.5 \mu \mathrm{g} / \mathrm{ml}$, the dose-response curve shows a strange trend (Figure 8). More specifically, as the dose increases there is a decrease in metabolic activity, as typically expected, until this critical point is reached. However, at concentrations above $12.5 \mu \mathrm{g} / \mathrm{ml}$, it appears that the metabolic activity begins to increase again. To overcome to the limitations of the colorimetric MTT assay for assessing Med-NPs' safety, we propose the use of HCS to monitor tetramethylrhodamine methyl ester (TMRM) accumulations in mitochondria with intact membrane potentials [86], to avoid the necessity for colorimetric tests. HCS has also previously been shown to be suitable for the assessment of NP cytotoxicity and cellular viability [80,87-89].

To advance the development of methods that could be applied for assessing toxicity of Med-NPs, we have suggested Hoechst and PI staining as an alternative to LDH release assay and Hoechst-TMRM as alternative to MTT. The detection of the staining can be accomplished through a variety of techniques including immunofluorescence microscopy, FACS or the HCS platform. Advanced technologies, such as HCS, have the advantage of providing reliable data in terms of statistics, and in parallel to its applications in assessing basic information on the toxicity of the Med-NPs, it could also indicate the interaction of the nanomaterial with the various cell compartments and contribute to the clarification of the mode of action of the drug candidate $[63,90,91]$. HCS also has the potential to be used in assessing immuno- and genotoxicity [80].

We are confident that methods that do not include colorimetric detection can overcome identified pitfalls of the classical standardized methods when applied to toxicity assessment of nanomaterials. The use of noncolorimetric 
assays can foster the identification of suitable in vitro cytotoxicity methods applicable for Med-NPs; methods that are urgently needed by the regulators to support Med-NPs' approval for clinical trials or market authorization.

\section{Conclusion \& future perspective}

This perspective aims to highlight the need to further develop and standardize methods for the accurate characterization and evaluation of nanomedicines, and especially for the next generation of nanomedicines. Our primary objective was to raise consciousness of the pitfalls of the current methods when applied to determine PSD, drug loading and in vitro cytotoxicity of Med-NPs, and second, to stimulate innovation through supporting the development of new analytical assays or by adapting existing methods, tools and standards.

The regulatory community will benefit from obtaining information on the performance of new or adapted tests, which could be applied to the current and next generation of nanomedicines as well as to nanosimilars.

Moreover, H2020 projects like EU-NCL and REFINE are established to foster the development and use and of protocols for the preclinical characterization of Med-NPs. Having reliable methods will be beneficial both for the preclinical stages of nanomedicine development and for supporting regulators in the assessment of quality and safety of Med-NPs.

To conclude, accelerating the development of the next generation of nanomedicines is a crucial step:

- To identify critical physicochemical parameters related to nanomaterial interactions with biological systems;

- Harmonization of standards, protocols and assays for Med-NPs;

- The development of widely available Med-NP-relevant reference standards for method validation purposes;

- The availability of competent multidisciplinary infrastructures (EU-NCL, NCI-NCL) to support Med-NP developers and regulatory needs.

\section{Executive summary}

- Rapid growth of nanomedicine products requires adaptation and development of new analysis toolkits and methodologies.

- Requirement for harmonization of protocols and methodologies to ensure approval of new nanomedicines.

Physicochemical characterization

- Accurate particle size and distribution data are a priority for researchers and regulators.

- Dynamic light scattering is widely used but has limitations when analyzing polydisperse samples.

- Nanoparticle tracking analysis can analyze polydisperse samples, but characterization can be hindered by the refractive index of the material.

- Filtration and fractionation of the sample prior to analysis by field flow fractionation can greatly improve the quality of the results, for example, when analyzing the medical nanoparticles' interactions with serum proteins.

Drug loading/drug release kinetics

- Knowledge of the quantity of drug loaded in a nanomedicine, and its release profile is critical.

- HPLC is the standard approach, but analytical ultracentrifugation may be better suited due to it combining separation, concentration and detection steps into one single measurement.

In vitro toxicity

- ISO and ASTM standards exist for the in vitro assessment of nanoparticle toxicity.

- Commonly used assays include LDH and MTT assays, but these assays have been shown to suffer from interference from the nanoparticle.

- Fluorescence-based approaches using high content screening can overcome the issue of nanoparticle absorbance in colorimetric assays.

Conclusion

- Pitfalls exist in the assessment of nanomedicine products and innovation and collaboration required to address these issues.

- Projects such as EU-NCL and REFINE act to develop and use protocols for preclinical characterization of nanomedicines to support developers and regulatory needs. 


\section{Acknowledgements}

The authors would like to thank P Boisseau for his contribution. They are very thankful to the EU-NCL and US-NCL consortium.

\section{Financial \& competing interests disclosure}

This research was partially carried out within the EU-NCL project, a European Union's Horizon 2020 Research Infrastructure under grant agreement no. 654190. This work was also supported by the European Commission's Joint Research Center within the Consumer Products Safety of the Directorate of Health, Consumers and Reference Materials through the Joint Research Center Multiannual Work Program. The authors have no other relevant affiliations or financial involvement with any organization or entity with a financial interest in or financial conflict with the subject matter or materials discussed in the manuscript apart from those disclosed.

No writing assistance was utilized in the production of this manuscript.

\section{References}

1 Sainz V, Conniot J, Matos AI et al. Regulatory aspects on nanomedicines. Biochem. Biophys. Res. Commun. 468(3), 504-510 (2015).

2 Pita R, Ehmann F, Papaluca M. Nanomedicines in the EU - regulatory overview. AAPS J. 18(6), 1576-1582 (2016).

3 Musazzi UM, Marini V, Casiraghi A, Minghetti P. Is the European regulatory framework sufficient to assure the safety of citizens using health products containing nanomaterials? Drug Discov. Today 22(6), 870-882 (2017).

4 IPRF.org - International Pharmaceutical Regulators Forum.www.i-p-r-f.org/index.php/en/

5 EU-NCL: European Nanomedicine Characterisation Laboratory.www.euncl.eu/

6 Us Food and Drug Administration. Global Summit on Regulatory Science (GSRS16). Presented at: Global Summit on Regulatory Science1 (GSRS16) Nanotechnology Standards and Applications. Bethesda, MD, USA, 7-9 September 2016.

7 Tyner KM, Zou P, Yang X, Zhang H, Cruz CN, Lee SL. Product quality for nanomaterials: current US experience and perspective. Wiley Interdisc. Rev. Nanomed. Nanobiotechnol. 7(5), 640-654 (2015).

8 D'mello SR, Cruz CN, Chen M-L, Kapoor M, Lee SL, Tyner KM. The evolving landscape of drug products containing nanomaterials in the United States. Nat. Nanotechnol. 12(6), 523-529 (2017).

9 De Jong WH, Hagens WI, Krystek P, Burger MC, Sips AJ, Geertsma RE. Particle size-dependent organ distribution of gold nanoparticles after intravenous administration. Biomaterials 29(12), 1912-1919 (2008).

10 Alexis F, Pridgen E, Molnar LK, Farokhzad OC. Factors affecting the clearance and biodistribution of polymeric nanoparticles. Mol. Pharm. 5(4), 505-515 (2008).

11 Mitragotri S, Lahann J. Physical approaches to biomaterial design. Nat. Mater. 8(1), 15 (2009).

12 ISO. Particle size analysis - dynamic light scattering (DLS) (ISO 22412:2017) (2017).

13 ISO. Particle size analysis - photon correlation spectroscopy (ISO 13321:1996) (1996).

14 ASTM. Standard guide for measurement of particle size distribution of nanomaterials in suspension by photon correlation spectroscopy (PCS) (E2490-09) (2015).

15 Varenne F, Makky A, Gaucher-Delmas M, Violleau F, Vauthier C. Multimodal dispersion of nanoparticles: a comprehensive evaluation of size distribution with nine size measurement methods. Pharm. Res. 33(5), 1220-1234 (2016).

16 Mehn D, Caputo F, Rösslein M et al. Larger or more? Nanoparticle characterisation methods for recognition of dimers. RSC Adv. 7(44), 27747-27754 (2017).

17 Anderson W, Kozak D, Coleman VA, Jämting ÅK, Trau M. A comparative study of submicron particle sizing platforms: accuracy, precision and resolution analysis of polydisperse particle size distributions. J. Colloid Interface Sci. 405, 322-330 (2013).

18 Measuring the NP stability against aggregation as a function of time and buffers with batch-mode DLS.www.euncl.eu/about-us/assay-cascade/PDFs/PCC/EUNCL-PCC-021.pdf?m=1468937870

19 Calzolai L, Gilliland D, Garcì CP, Rossi F. Separation and characterization of gold nanoparticle mixtures by flow-field-flow fractionation. J. Chromatogr. 1218(27), 4234-4239 (2011).

20 Hondow N, Brydson R, Wang P et al. Quantitative characterization of nanoparticle agglomeration within biological media. J. Nanopart. Res. 14(7), 977 (2012).

21 Maguire CM, Sillence K, Roesslein M et al. Benchmark of nanoparticle tracking analysis on measuring nanoparticle sizing and concentration. J. Micro. Nanomanuf. 5(4), 041002 (2017).

22 Walker JG. Improved nanoparticle tracking analysis. Meas. Sci. Technol. 23(6), 065605 (2012).

23 Iavicoli P, Urbán P, Bella A, Ryadnov MG, Rossi F, Calzolai L. Application of asymmetric flow field-flow fractionation hyphenations for liposome-antimicrobial peptide interaction. J. Chromatogr. 1422, 260-269 (2015).

24 Ingallina C, Rinaldi F, Bogni A et al. Niosomal approach to brain delivery: development, characterization and in vitro toxicological studies. Int. J. Pharm. 511(2), 969-982 (2016). 
25 Hole P, Sillence K, Hannell C et al. Interlaboratory comparison of size measurements on nanoparticles using nanoparticle tracking analysis (NTA). J. Nanopart. Res. 15(12), 1-12 (2013).

26 Yohannes G, Jussila M, Hartonen K, Riekkola M-L. Asymmetrical flow field-flow fractionation technique for separation and characterization of biopolymers and bioparticles. J. Chromatogr. 1218(27), 4104-4116 (2011).

27 Wagner M, Holzschuh S, Traeger A, Fahr A, Schubert US. Asymmetric flow field-flow fractionation in the field of nanomedicine. Anal. Chem. 86(11), 5201-5210 (2014).

28 Schädlich A, Rose C, Kuntsche J et al. How stealthy are PEG-PLA nanoparticles? An NIR in vivo study combined with detailed size measurements. Pharm. Res. 28(8), 1995-2007 (2011).

29 Spadavecchia J, Movia D, Moore C et al. Targeted polyethylene glycol gold nanoparticles for the treatment of pancreatic cancer: from synthesis to proof-of-concept in vitro studies. Int. J. Nanomedicine 11, 791-822 (2016).

30 Measurement of particle size distribution of protein binding, of mean molecular weight of polymeric NP components, study of batch to batch reproducibility and study of release of free coating from NP surface by FFF-MALS.www.euncl.eu/about-us/assay-cascade/PDFs/PCC/EUNCL-PCC-022.pdf?m=1468937868

31 Miller T, Rachel R, Besheer A, Uezguen S, Weigandt M, Goepferich A. Comparative investigations on in vitro serum stability of polymeric micelle formulations. Pharm. Res. 29(2), 448-459 (2012).

32 Astm. Standard guide for measurement of particle size distribution of nanomaterials in suspension by nanoparticle tracking analysis (NTA) (E2834-12) (2012).

33 Iso. Particle size analysis - particle tracking analysis (PTA) method (ISO 19430:2016) (2016).

34 Filipe V, Hawe A, Jiskoot W. Critical evaluation of nanoparticle tracking analysis (NTA) by NanoSight for the measurement of nanoparticles and protein aggregates. Pharm. Res. 27(5), 796-810 (2010).

35 Williams SKR, Runyon JR, Ashames AA. Field-flow fractionation: addressing the nano challenge. Anal. Chem. 83(3), 634-642 (2010).

36 Contado C. Field flow fractionation techniques to explore the 'nano-world'. Anal. Bioanal. Chem. 409(10), 2501-2518 (2017).

37 Zattoni A, Roda B, Borghi F, Marassi V, Reschiglian P. Flow field-flow fractionation for the analysis of nanoparticles used in drug delivery. J. Pharm. Biomed. Anal. 87, 53-61 (2014).

38 EU-NCL Assay Cascade.www.euncl.eu/about-us/assay-cascade/

39 Barenholz YC. Doxil ${ }^{\circledR}$ - the first FDA-approved nanodrug: lessons learned. J. Control. Release 160(2), 117-134 (2012).

40 Delmas T, Couffin A-C, Bayle PA et al. Preparation and characterization of highly stable lipid nanoparticles with amorphous core of tuneable viscosity. J. Colloid Interface Sci. 360(2), 471-481 (2011).

41 Committee for Medicinal Products for Human Use. Joint MHLW/EMA reflection paper on the development of block copolymer micelle medicinal products (2013).www.ema.europa.eu/docs/en_GB/document_library/Scientific_guideline/2013/02/WC500138390.pdf

42 Committee for Medicinal Products for Human Use. Reflection paper on the data requirements for intravenous liposomal products developed with reference to an innovator liposomal product (2013).www.ema.europa.eu/docs/en_GB/document_library/Scientific_guideline/2013/03/WC500140351.pdf

43 The European Parliament and the Council of the European Union. Directive 2001/83/EC of the European parliament and of the council of 6 November 2001 on the community code relating to medicinal products for human use. Official Journal of the European Commission (L 311/67), 67-128 (2001).

44 U.S. Department of Health and Human Services. Analytical procedures and methods validation for drugs and biologics - guidance for industry. (2015).www.fda.gov/downloads/drugs/guidances/ucm386366.pdf

45 Committee for proprietary medicinal products ICH Q2 (R1) validation of analytical procedures: text and methodology. (1995).www.ich.org/products/guidelines/quality/quality-single/article/validation-of-analytical-procedures-text-and-methodology.html

46 Edwards KA, Baeumner AJ. Analysis of liposomes. Talanta 68(5), 1432-1441 (2006).

47 Gómez-Hens A, Fernández-Romero JM. Analytical methods for the control of liposomal delivery systems. Trends Anal. Chem. 25(2), 167-178 (2006).

48 Chimanuka B, Gabriels M, Detaevernier MR, Plaizier-Vercammen JA. Preparation of beta-artemether liposomes, their HPLC-UV evaluation and relevance for clearing recrudescent parasitaemia in Plasmodium chabaudi malaria-infected mice. J. Pharm. Biomed. Anal. 28(1), 13-22 (2002).

49 Wu P-C, Tsai Y-H, Liao C-C, Chang J-S, Huang Y-B. The characterization and biodistribution of cefoxitin-loaded liposomes. Int. J. Pharm. 271(1), 31-39 (2004).

50 Junping W, Maitani Y, Takayama K, Nagai T. In vivo evaluation of doxorubicin carried with long circulating and remote loading proliposome. Int. J. Pharm. 203(1-2), 61-69 (2000).

51 Bellott R, Pouna P, Robert J. Separation and determination of liposomal and nonliposomal daunorubicin from the plasma of patients treated with Daunoxome. J. Chromatogr. B Biomed. Sci. Appl. 757(2), 257-267 (2001). 
52 Mehn D, Iavicoli P, Cabaleiro N et al. Analytical ultracentrifugation for analysis of doxorubicin loaded liposomes. Int. J. Pharm. 523(1), 320-326 (2017).

53 Brown PH, Schuck P. Macromolecular size-and-shape distributions by sedimentation velocity analytical ultracentrifugation. Biophys. J. 90(12), 4651-4661 (2006).

54 Dam J, Velikovsky CA, Mariuzza RA, Urbanke C, Schuck P. Sedimentation velocity analysis of heterogeneous protein-protein interactions: Lamm equation modeling and sedimentation coefficient distributions c(s). Biophys. J. 89(1), 619-634 (2005).

55 Schuck P. Size-distribution analysis of macromolecules by sedimentation velocity ultracentrifugation and lamm equation modeling. Biophys. J. 78(3), 1606-1619 (2000).

56 Carney RP, Kim JY, Qian H et al. Determination of nanoparticle size distribution together with density or molecular weight by 2D analytical ultracentrifugation. Nat. Commun. 2, 335 (2011).

57 Wohlleben W. Validity range of centrifuges for the regulation of nanomaterials: from classification to as-tested coronas. J. Nanopart. Res. 14(12), 1300 (2012).

58 Mittal V, Volkel A, Colfen H. Analytical ultracentrifugation of model nanoparticles: comparison of different analysis methods. Macromol. Biosci. 10(7), 754-762 (2010).

59 Planken KL, Colfen H. Analytical ultracentrifugation of colloids. Nanoscale 2(10), 1849-1869 (2010).

60 Görög S. The sacred cow: the questionable role of assay methods in characterising the quality of bulk pharmaceuticals. J. Pharm. Biomed. Anal. 36(5), 931-937 (2005).

61 Doke SK, Dhawale SC. Alternatives to animal testing: a review. Saudi Pharm. J. 23(3), 223-229 (2015).

62 Oecd. Draft guidance document on good in vitro method practices (Givimp) for the development and implementation of in vitro methods for regulatory use in human safety assessment (2016).www.oecd.org/env/ehs/testing/OECD_Draft_GIVIMP_in_Human_Safety_Assessment.pdf

63 Zanella F, Lorens JB, Link W. High content screening: seeing is believing. Trends Biotechnol. 28(5), 237-245 (2010).

64 Damoiseaux R, George S, Li M et al. No time to lose - high-throughput screening to assess nanomaterial safety. Nanoscale 3(4), 1345-1360 (2011).

65 Beger RD, Sun J, Schnackenberg LK. Metabolomics approaches for discovering biomarkers of drug-induced hepatotoxicity and nephrotoxicity. Toxicol. Appl. Pharmacol. 243(2), 154-166 (2010).

66 Matthews H, Hanison J, Nirmalan N. 'Omics' - informed drug and biomarker discovery: opportunities, challenges and future perspectives. Proteomes 4(3), 28 (2016).

67 Niles AL, Moravec RA, Riss TL. In vitro viability and cytotoxicity testing and same-well multiparametric combinations for high-throughput screening. Curr. Chem. Genomics 3, 33 (2009).

68 Bregoli L, Movia D, Gavigan-Imedio JD, Lysaght J, Reynolds J, Prina-Mello A. Nanomedicine applied to translational oncology: a future perspective on cancer treatment. Nanomed. Nanotechnol. Biol. Med. 12(1), 81-103 (2016).

69 Waxenecker G, Binder R. Regulatory animal testing for the development of medicines. In: Comparative Medicine. Springer, 209-218 (2017). Guhad F. Introduction to the 3Rs (refinement, reduction and replacement). J. Am. Assoc. Lab. Anim. Sci. 44(2), 58-59 (2005).

71 Wells DJ. Animal welfare and the 3Rs in European biomedical research. Ann. NY Acad. Sci. 1245(1), 14-16 (2011).

72 ISO. Biological evaluation of medical devices (ISO 10993:2009). ISO 10993-5:2009 (2009).

73 Astm. Standard test method for evaluation of cytotoxicity of nanoparticulate materials in porcine kidney cells and human hepatocarcinoma cells (E2526-08) (2013).

74 Wörle-Knirsch J, Pulskamp K, Krug H. Oops they did it again! Carbon nanotubes hoax scientists in viability assays. Nano Lett. 6(6), 1261-1268 (2006).

75 Belyanskaya L, Manser P, Spohn P, Bruinink A, Wick P. The reliability and limits of the MTT reduction assay for carbon nanotubes-cell interaction. Carbon 45(13), 2643-2648 (2007).

76 Guadagnini R, Halamoda Kenzaoui B, Walker L et al. Toxicity screenings of nanomaterials: challenges due to interference with assay processes and components of classic in vitro tests. Nanotoxicology 9(Suppl. 1), 13-24 (2015).

77 Han X, Gelein R, Corson N et al. Validation of an LDH assay for assessing nanoparticle toxicity. Toxicology 287(1), 99-104 (2011).

78 Monteiro-Riviere N, Inman A, Zhang L. Limitations and relative utility of screening assays to assess engineered nanoparticle toxicity in a human cell line. Toxicol. Appl. Pharmacol. 234(2), 222-235 (2009).

79 Casey A, Herzog E, Davoren M, Lyng F, Byrne H, Chambers G. Spectroscopic analysis confirms the interactions between single walled carbon nanotubes and various dyes commonly used to assess cytotoxicity. Carbon 45(7), 1425-1432 (2007).

80 Collins AR, Annangi B, Rubio L et al. High-throughput toxicity screening and intracellular detection of nanomaterials. Wiley Interdisciplin. Rev. Nanomed. Nanobiotechnol. 9(1), doi:10.1002/wnan.1413 (2017) (Epub ahead of print). 
81 ISO. Biological evaluation of medical devices - Part 22: guidance on nanomaterials (ISO/TR 10993-22). ISO/TR 10993-22:2017 (2017).

82 Bremer S, Halamoda-Kenzaoui B, Borgos SE. Identification of regulatory needs for nanomedicines 1st EU-NCL survey with the 'Nanomedicine' working group of the international pharmaceutical regulators. Publications Office of the European Union EUR 28224 EN (2016).

83 Oh S-J, Kim H, Liu Y et al. Incompatibility of silver nanoparticles with lactate dehydrogenase leakage assay for cellular viability test is attributed to protein binding and reactive oxygen species generation. Toxicol. Lett. 225(3), 422-432 (2014).

84 Ong KJ, Maccormack TJ, Clark RJ et al. Widespread nanoparticle-assay interference: implications for nanotoxicity testing. PLoS ONE 9(3), e90650 (2014).

85 Lupu A, Popescu T. The noncellular reduction of MTT tetrazolium salt by $\mathrm{TiO}_{2}$ nanoparticles and its implications for cytotoxicity assays. Toxicol. In vitro 27(5), 1445-1450 (2013).

86 Scaduto RC, Grotyohann LW. Measurement of mitochondrial membrane potential using fluorescent rhodamine derivatives. Biophys. J. 76(1), 469-477 (1999).

87 Byrne F, Prina-Mello A, Whelan A et al. High content analysis of the biocompatibility of nickel nanowires. J. Magn. Magn. Mater. 321(10), 1341-1345 (2009).

88 Movia D, Gerard V, Maguire CM et al. A safe-by-design approach to the development of gold nanoboxes as carriers for internalization into cancer cells. Biomaterials 35(9), 2543-2557 (2014).

89 Kuznetsova VA, Visheratina AK, Ryan A et al. Enantioselective cytotoxicity of ZnS:Mn quantum dots in A549 cells. Chirality 29(8), 403-408 (2017).

90 Lang P, Yeow K, Nichols A, Scheer A. Cellular imaging in drug discovery. Nat. Rev. Drug Discov. 5(4), 343-356 (2006).

91 Bickle M. The beautiful cell: high-content screening in drug discovery. Anal. Bioanal. Chem. 398(1), 219-226 (2010). 